\title{
Sensor Placement Optimization of Vibration Test on Medium-Speed Mill
}

\author{
Lihua Zhu, ${ }^{1,2}$ Jun Dai, ${ }^{1}$ and Guoliang Bai ${ }^{1}$ \\ ${ }^{1}$ School of Civil Engineering, Xian University of Architecture and Technology, Xian 710055, China \\ ${ }^{2}$ Department of Civil, Structural and Environmental Engineering, University at Buffalo, State University of New York, \\ Buffalo, NY 14260, USA \\ Correspondence should be addressed to Lihua Zhu; zhulihuaxa@163.com
}

Received 8 September 2014; Revised 22 December 2014; Accepted 24 December 2014

Academic Editor: Stathis C. Stiros

Copyright (C) 2015 Lihua Zhu et al. This is an open access article distributed under the Creative Commons Attribution License, which permits unrestricted use, distribution, and reproduction in any medium, provided the original work is properly cited.

\begin{abstract}
Condition assessment and decision making are important tasks of vibration test on dynamic machines, and the accuracy of dynamic response can be achieved by the sensors placed on the structure reasonably. The common methods and evaluation criteria of optimal sensor placement (OSP) were summarized. In order to test the vibration characteristic of medium-speed mill in the thermal power plants, the optimal placement of 12 candidate measuring points in $X, Y$, and $Z$ directions on the mill was discussed for different targeted modal shapes, respectively. The OSP of medium-speed mill was conducted using the effective independence method (EfI) and QR decomposition algorithm. The results showed that the order of modal shapes had an important influence on the optimization results. The difference of these two methods on the sensor placement optimization became smaller with the decrease of the number of target modes. The final scheme of OSP was determined based on the optimal results and the actual test requirements. The field test results were basically consistent with the finite element analysis results, which indicated the sensor placement optimization for vibration test on the medium-speed mill was feasible.
\end{abstract}

\section{Introduction}

The model updating, condition identification, condition assessment, and decision making of the concerned structure require enough efficient data to reflect structural properties. It is a common way to achieve the related data by sensors. However, restricted by testing technology and cost, a practical problem is how to select a set with a minimum number of sensor locations from all possibilities [1]. Optimal sensor placement (OSP) has been one of the more important tasks in dynamic test, especially for those high-rise buildings and complicated structures.

The basic principle of sensor placement optimization is that limited measuring points can get enough efficient data which can reflect the structural properties. Recently, most methods aim to achieve the best identification of structural characteristics, including the frequencies and mode shapes. Among these methods, effective independence (EfI) method is the most widely used method, which was proposed by Kammer [2]. For modal kinetic method (MKE), the target of
OSP is to ensure the maximization of the measured kinetic mode energy that improves signal-to-noise ratio [3]. Besides, EfI is an iterated version of the MKE with reorthonormalized mode shapes [4]. The modal assurance criterion (MAC) method is based on $\mathrm{QR}$ decomposition with column pivoting, and these measuring points are chosen to minimize offdiagonal element of MAC matrix [5].

So far, a multitude of researches have been conducted on sensor placement optimization for large-span bridges and high-rise buildings in civil engineering. Yi et al. conducted sensor placement optimization for Dalian International Trade Mansion [1]. Chow et al. presented optimal sensor configuration of a typical transmission tower for the purpose of structural model updating [6]. Wang et al. established the wind and structural health monitoring system implemented on the Runyang Yangtze River Bridge [7]. However, there are few researches on the sensor placement optimization for those structures which are not very high or large, while the vibration modes are very complicated. The medium-speed mill is one of the most commonly used auxiliary power items 
of equipment in thermal power plants. It generates significant disturbing force when it is running, so it is very important to monitor its vibration condition. The study of Zhu et al. shows that vibration modes of medium-speed mill are very complicated [8].

In order to obtain the reliable dynamic response, reasonable condition assessment, and decision making of mediumspeed mill, the measuring points should be placed on the locations that reflect abundant structural information of medium-speed mill. So the OSP for vibration test on medium-speed mill is extremely necessary. In this paper, a reliable finite element model (FEM) of medium-speed mill supported by spring vibration-isolated foundation is built, and two OSP methods are applied to the vibration test of medium-speed mill. Finally, considering the actual situation of vibration test, an OSP scheme is proposed in the vibration test on medium-speed mill.

\section{The Algorithm and Evaluation Criteria of OSP}

2.1. Effective Independence Method. The aim of the EfI method is to select measurement positions that make the mode shapes of interest as linearly independent as possible while containing sufficient information about the target modal responses in the measurements.

The measured structural response can be expressed as

$$
\mathbf{u}=\boldsymbol{\Phi} \mathbf{q}+\mathbf{w}=\sum_{i=1}^{N} q_{i} \boldsymbol{\varphi}_{i}+\mathbf{w},
$$

where $\boldsymbol{\Phi}$ is the matrix of FEM target mode shapes, $\mathbf{q}$ is the coefficient response vector and also is modal coordinate, and $\mathbf{w}$ is a sensor noise vector, assumed stationary random process with a mean value zero. There must be a deviation for corresponding real generalized coordinate $\widehat{\mathbf{q}}$. Assuming that this process is an effective unbiased estimation, the covariance matrix $\mathbf{J}$ is obtained as

$$
\mathbf{J}=E\left[(\mathbf{q}-\widehat{\mathbf{q}})(\mathbf{q}-\widehat{\mathbf{q}})^{T}\right]=\left[\boldsymbol{\sigma}^{-2} \boldsymbol{\Phi}^{T} \boldsymbol{\Phi}\right]^{-1}=\mathbf{Q}^{-1},
$$

where $\mathbf{Q}$ is the Fisher information matrix (FIM) by assuming the measured noise is independent and has the same statistical properties. The matrix $\mathbf{Q}$ can be written as

$$
\mathbf{Q}=\boldsymbol{\sigma}^{-2} \boldsymbol{\Phi}^{T} \boldsymbol{\Phi}=\boldsymbol{\sigma}^{-2} \mathbf{A}
$$

Then, the maximization of $\mathbf{Q}$ is equivalent to the maximization of $\mathbf{A}$, and thus $\mathbf{A}$ can be used to simplify FIM. Constructing matrix $\mathbf{E}_{D}$,

$$
\mathbf{E}_{D}=\boldsymbol{\Phi}\left[\boldsymbol{\Phi}^{T} \boldsymbol{\Phi}\right]^{-1} \boldsymbol{\Phi}^{T}
$$

where $\mathbf{E}_{D}$ is the effective independence allocation matrix. The diagonal elements of the $\mathbf{E}_{D}$ represent the contribution of the candidate points to the modal matrix linearly independent.

2.2. The Algorithm for OSP Based on QR Decomposition. $\mathrm{QR}$ decomposition is derived from the maximization of the
FIM. The linearly independent row vectors are extracted from modal matrix by QR decomposition that ensures a larger norm of matrix. These degrees of freedom (DOFs) corresponding with linearly independent row vectors can be used as an ideal placement for sensors. The decomposition can be expressed as

$$
\mathbf{A}=\boldsymbol{\Phi}^{T} \mathbf{E}=\mathbf{Q R}
$$

where $\mathbf{E}$ is the unit conversion matrix, descending order according to the value of the diagonal elements of $\mathbf{R}$. We choose the modal DOFs in accordance with the sequence of the elements in $\mathbf{E}$.

\subsection{The Evaluation Criteria of OSP}

2.3.1. Fisher Information Matrix Determinant. According to the principle of EfI method, the greater the trace or the value of FIM determinant is, the smaller the covariance $\mathbf{J}$ is and the more effective the modal coordinate is. Meanwhile, FIM also measures the abundance modal information from the response of structures, and the greater the determinant value is, the more abundant modal information it contains [5].

2.3.2. Modal Assurance Criterion. The quantity of measured DOFs is far less than the whole DOFs of structural model and the responses are affected by measurement accuracy and noise; thus, the modal vector measured cannot retain the original space property. MAC is a good method to evaluate the space angle of modal vectors [9]. The formula is expressed as

$$
\mathrm{MAC}_{i j}=\frac{\left(\phi_{i}^{T} \phi_{j}\right)^{2}}{\left(\phi_{i}^{T} \phi_{i}\right)\left(\phi_{j}^{T} \phi_{j}\right)}
$$

where $\phi_{i}$ and $\phi_{j}$ are the $i$ th and $j$ th modal vectors, respectively. The off-diagonal elements of MAC matrix should be minimized for OSP, and thus the modal matrix measured can keep better orthogonality.

\section{OSP for Vibration Test on Medium-Speed Mill}

3.1. Description of the Medium-Speed Mill. Medium-speed mill is an important auxiliary dynamic machine in the thermal power plants. To mitigate the vibration, the spring vibration-isolated foundation is normally used to support the mill. A spring vibration-isolated foundation for mediumspeed mill is typically comprised of upper and lower bedplates and spring isolators. An elevation view of a vibration-isolated foundation for medium-speed mill is shown in Figure 1. As shown, there are two buttresses over the lower bedplate, and the distance between the upper bedplate and buttresses is $330 \mathrm{~mm}$. Spring isolators are placed between the two buttresses and the upper bedplate. Twelve sets of spring isolators are placed on each buttress; thus, a total of 24 sets of isolators are placed. 


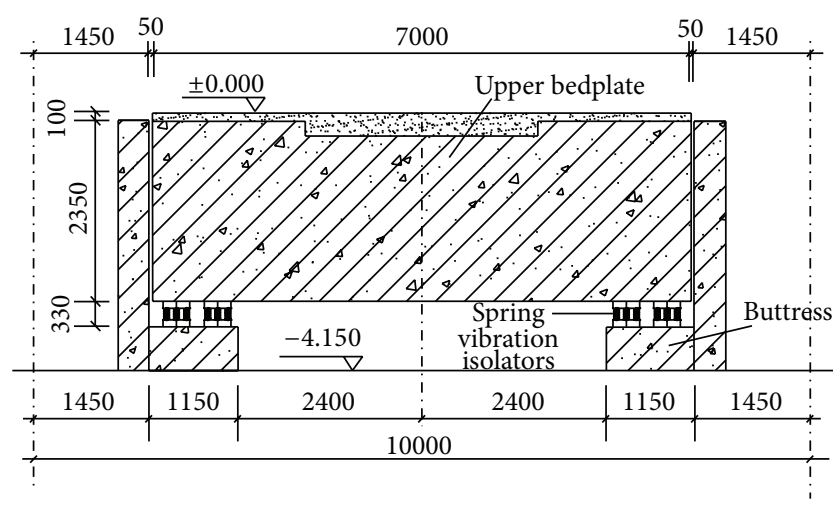

FIGURE 1: Spring vibration-isolated foundation of medium-speed mill.

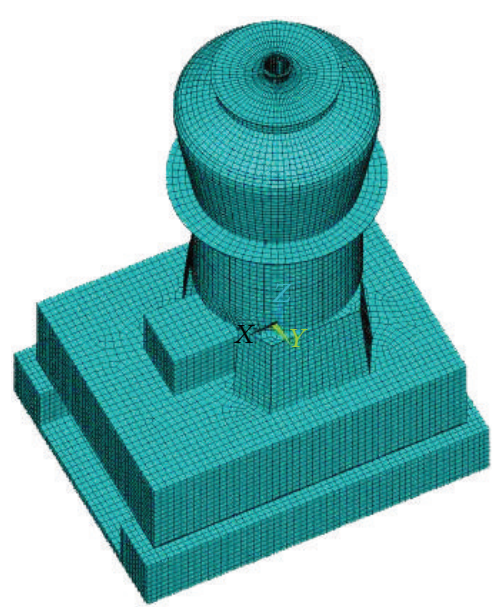

FIgURE 2: Finite element model.

\subsection{Finite Element Analysis}

3.2.1. Modeling. A medium-speed mill in the Yuzhou power plant (Henan Province, China) was used as the prototype for the developed finite element model. In order to provide the input data for the optimization analysis, a three-dimensional finite element coupled model of a medium-speed mill and its foundation was built using ANSYS (version 12.0) [10]. SOLID185 elements were used to simulate the foundation, the mill, and the motor; SHELL181 elements were used for the frame and chassis; BEAM188 elements were used for the pressure frame; COMBINE14 elements were used for the isolators; and MASS21 elements were used for the load.

The frame, motor, and mill were rigidly connected with the upper bedplate of the foundation. The boundary condition of the lower bedplate of the foundation was simulated using spring elements, which represented the constraint effect of the soil on the foundation. For the coordinate system of the model, $X$ represented the transverse direction of the foundation of medium-speed mill and $Y$ represented the longitudinal direction. The mesh size of the finite element model was between 100 and $200 \mathrm{~mm}$. The finite element model was shown in Figure 2, and the primary parameters of the mill and vibration isolator were listed in Table 1.
3.2.2. Modal Analysis. After building a FE model of the medium-speed mill with spring vibration-isolated foundation, a modal analysis was done using the block Lanczos method. The vibration modal shapes and corresponding frequencies were shown in Figure 3 and Table 2.

3.3. Selection of Candidate Measuring Points. The mediumspeed mill is an axial symmetric structure. According to ANSYS analysis, the primary vibration mode shapes of medium-speed mill include horizontal longitudinal vibration, horizontal transverse vibration, and vertical vibration. And also considering the cost and technical factors, measuring points were placed along one side of the medium-speed mill. 12 measuring points were set as candidate points. Each point had DOFs of $X, Y$, and $Z$ direction; thus, there were a total of 36 DOFs. EfI and QR methods were employed to optimize the sensors placement of medium-speed mill and the first 3, 4, 5, and 6 were taken as the monitoring modal shapes, respectively. The placement of candidate measuring points was shown in Figure 4.

36 DOFs were analyzed preliminarily using EfI method with MATLAB software. According to $\mathbf{E}_{D}$, the contribution of 12 DOFs in $Z$ direction to modal matrix linear independence was far less. There is no sufficient reason to believe that the FE model can simulate the actual structure accurately, so removing the DOFs in $Z$ direction just based on the optimization results of 36 DOFs, the vibration characteristic of medium-speed mill may not be understood comprehensively. Motivated by the above consideration, it is determined to optimize the placement of 12 DOFs in $X, Y$, and $Z$ direction, respectively.

3.4. Results of OSP. The OSP of 12 candidate measuring points was conducted using EfI method and QR decomposition algorithm for different target mode shapes in $X, Y$, and $Z$ direction, respectively, and the results of OSP were presented in Table 3. It was noted that those measuring points in brackets were suboptimal ones. The suboptimal measuring points were not included in the optimization results in this paper, while the referred optimization results meant that the number of measuring points was consistent with the number of target mode shapes.

In Table 3, it was found that two optimization results did not agree with each other exactly. The optimization results of the first 3 and 4 mode shapes in $X$ direction were consistent exactly, but they were different for one and two measuring points in the first 5 and 6 mode shapes, respectively. The optimization results in $X$ direction had the trend that the fewer the number of target mode shapes was, the smaller the difference in two results was. Although the same trend did not appear in $Y$ and $Z$ directions, the optimization results were still consistent basically. Therefore, EfI and QR decomposition algorithms in OSP for medium-speed mill were feasible.

FIM determinant behavior was recorded in terms of percentage of its initial value against the decrease in candidate measuring points [11], and the results were shown in Figure 5. It was noted that the FIM determinant in lower orders of target mode shapes had larger value in case of the 
TABLE 1: Parameters of the mill and vibration isolator.

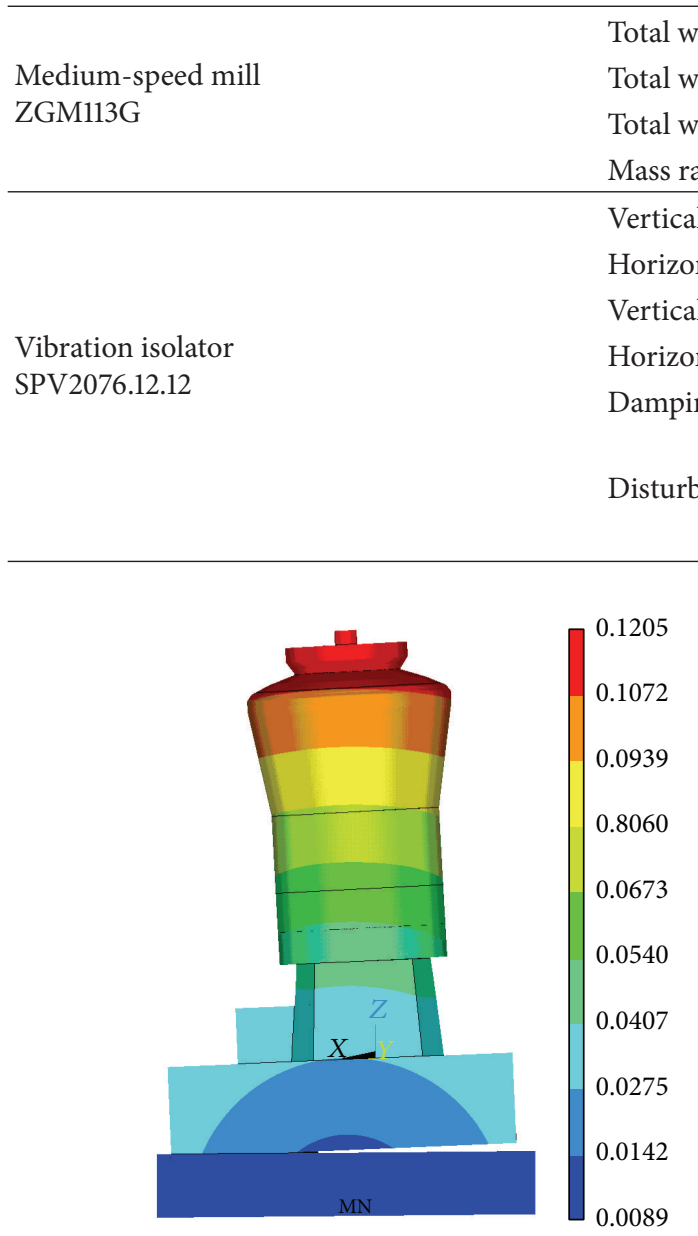

(a) First modal shape
Total weight of the mill/t

weight of the foundation/t 157.40

398.74

556.14

2.53

12.10

9.50

240

160

0.02

$X$ direction $=10$

$Y$ direction $=10$

$Z$ direction $=40$

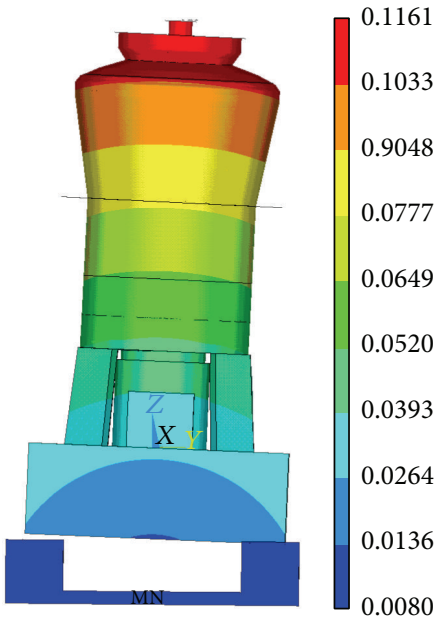

(b) Second modal shape

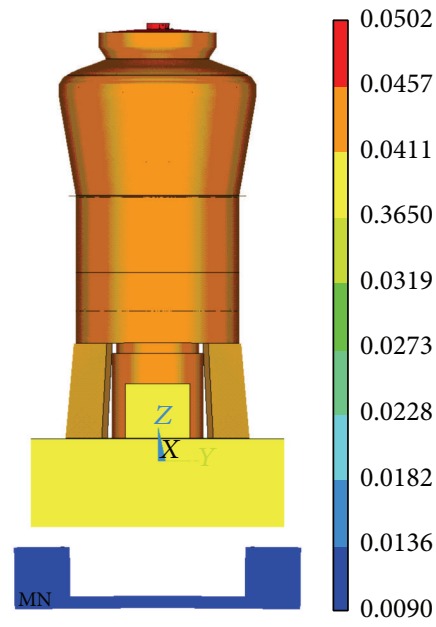

(c) Third modal shape

FIGURE 3: First three modes.

TABLE 2: Vibration frequencies.

\begin{tabular}{lll}
\hline Order & $\begin{array}{c}\text { Frequency } \\
f / \mathrm{Hz}\end{array}$ & Vibration modal shape \\
\hline 1 & 1.828 & $X$ direction horizontal vibration \\
2 & 2.003 & $Y$ direction horizontal vibration \\
3 & 3.578 & Vertical vibration \\
4 & 4.078 & Rotation around horizontal axis of the mill \\
5 & 4.235 & Torsion around the vertical axis \\
6 & 4.422 & Horizontal oblique translational vibration \\
\hline
\end{tabular}

same number of measuring points, which meant that these measuring points were more efficient in getting concerned information of the medium-speed mill. On the other hand, the value of FIM determinant increased more significantly when adding a measuring point in case of the same number of measuring points, which meant that the optimization results in larger orders were more sensitive to the number of measuring points. So the target modal played a significant role in the final scheme of the OSP.
The average values of off-diagonal elements of MAC matrix were shown in Figure 6. The average values of offdiagonal elements of MAC matrix were greater even if when 12 candidate measuring points were all placed. It was explained that the vibration modes of medium-speed mill were very complicated and even 12 measuring points cannot capture its dynamic characteristics expectedly. But the average value of off-diagonal elements of MAC increased a little in $Y$ direction using these two optimal methods to decrease the number of measuring points, while the average values in $X$ and $Z$ directions decreased, in particular in $Z$ direction. In other words, the expected optimal results could be achieved. Meanwhile, it could be confirmed that EfI method was much better due to smaller average values of MAC.

It was easy to find that the selection of suboptimal measuring points using two methods was significantly different. The values of FIM determinant after adding the suboptimal points to the optimization results and the final results were shown in Table 4. The value of FIM using EfI method was greater than that of $\mathrm{QR}$ decomposition method. The 


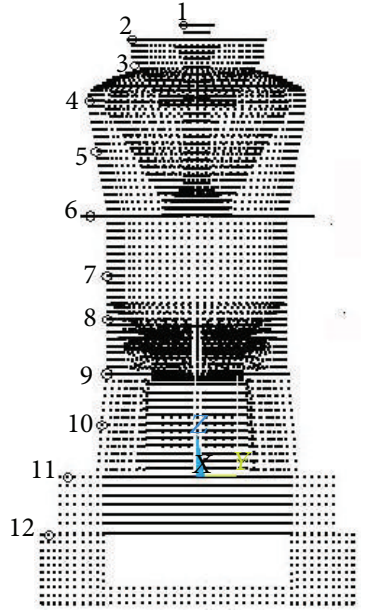

FIGURE 4: Placement of candidate measuring points.

TABLE 3: Optimization results of measuring points.

\begin{tabular}{|c|c|c|c|c|c|c|}
\hline \multirow{2}{*}{ Order } & \multicolumn{6}{|c|}{ Direction } \\
\hline & $X(\mathrm{EfI})$ & $X(\mathrm{QR})$ & $Y(\mathrm{EfI})$ & $Y(\mathrm{QR})$ & $Z$ (EfI) & $Z(\mathrm{QR})$ \\
\hline \multirow{6}{*}{6} & 1 & 1 & 3 & 1 & 1 & 1 \\
\hline & 4 & 4 & 4 & 4 & 2 & 2 \\
\hline & 7 & 8 & 5 & 5 & 4 & 6 \\
\hline & 8 & 9 & 9 & 9 & 5 & 7 \\
\hline & 9 & 10 & 10 & 10 & 10 & 10 \\
\hline & 11 & 11 & 11 & 11 & 11 & 11 \\
\hline \multirow{6}{*}{5} & 1 & 1 & 3 & 1 & 1 & 1 \\
\hline & 4 & 4 & 4 & 4 & 2 & 2 \\
\hline & 8 & 9 & 5 & 5 & 6 & 6 \\
\hline & 9 & 10 & 10 & 10 & 10 & 10 \\
\hline & 11 & 11 & 11 & 11 & 11 & 11 \\
\hline & (7) & (2) & (7) & (2) & (4) & (8) \\
\hline \multirow{6}{*}{4} & 1 & 1 & 3 & 1 & 1 & 1 \\
\hline & 4 & 4 & 4 & 4 & 4 & 6 \\
\hline & 10 & 10 & 7 & 7 & 10 & 10 \\
\hline & 11 & 11 & 10 & 10 & 11 & 11 \\
\hline & (9) & (8) & (1) & (8) & (6) & (8) \\
\hline & (6) & (2) & (5) & (2) & (9) & (2) \\
\hline \multirow{6}{*}{3} & 1 & 1 & 3 & 1 & 1 & 1 \\
\hline & 6 & 6 & 4 & 4 & 10 & 6 \\
\hline & 11 & 11 & 10 & 10 & 11 & 10 \\
\hline & (5) & (7) & (1) & (7) & (6) & (7) \\
\hline & (3) & (8) & (2) & (8) & (9) & (8) \\
\hline & (4) & (2) & (5) & (2) & (4) & (2) \\
\hline
\end{tabular}

effect of optimization using EfI method was better, and the corresponding measuring points had a stronger ability to get the information of the medium-speed mill.

\section{The Experimental Results of OSP}

4.1. Final Scheme of OSP. Based on the analysis of Section 3.2.2, the $X$-translational vibration was the primary vibration mode of medium-speed mill, so the optimal results of $X$ direction in the first 3 mode shapes using EfI method were employed. Considering the stability of data and security of sensors, the measuring point 1 was changed to point 2 , and they had the same testing condition. In addition, the data collected by the measuring point 2 was an important indicator to decide the working state of medium-speed mill. In order to reduce the effects of environmental and operational variability and noise contamination, the measuring points 2 and 6 with more modal kinetic energy were selected to improve the signal-to-noise ratio [3, 12-14]. The analysis of OSP showed that the contribution of the actual measuring point 12 in $X$, $Y$, and $Z$ directions is minimal, and it should be removed in theory. Considering that one of the aims of vibration test is to understand the isolation effect of the spring vibrationisolated foundation, the measuring point 12 was retained. From Table 3, the contribution of the measuring point 11 on the vibration of medium-speed mill in three directions was great, and the vertical vibration of medium-speed mill also should be tested. Therefore, the measuring points 2, 6, 11, and 12 were chosen as the final scheme of OSP.

In order to place the sensors in site easily, the measuring points were renumbered, which were shown in Figure 7. Among these test points, the HTP1 sensor was used to measure the horizontal vibration of the lower bedplate of the foundation or ground; the HTP2 sensor was attached to the upper bedplate; the HTP3 sensor was attached to the middle of the mill; and the HTP4 sensor was attached to the top of the mill. The VTP1 sensor was used to measure the vertical vibration of the lower bedplate of the foundation or ground; and the VTP2 sensor was attached to the upper bedplate of the foundation.

4.2. Field Tests. In order to verify the feasibility of OSP results, the medium-speed mill in the Yuzhou thermal power plant was tested in site. In the field test, 891-II type vibration measurement instruments including six sensors, amplifiers with a frequency response ranging from $0.5 \mathrm{~Hz}$ to $100 \mathrm{~Hz}$, an INV306 intelligent signal acquisition and processing analysis system, and a DASP data processing program were used. The sensors were placed according to the final scheme of OSP. The sensors used in the experiment were manufactured by the Institute of Engineering Mechanics, China Earthquake Administration. The sensors have four gears, which are used to test the large displacement, small displacement, velocity, and acceleration. Actually, they are displacement sensors. In this test, the gear of small displacement was used. The elevation view of the tested medium-speed mill was shown in Figure 8, and the sensor in close-up was shown in Figure 9.

The vibration test on the medium-speed mill was conducted in the plant, so the variability of physical property with thermal effect can be ignored [13]. The primary noise contamination was sourced from other nearby dynamic machines. The vibration data was recorded when the medium-speed mill did not run, which was used in the next filtering [12].

Field tests were conducted during the working steady stage of the medium-speed mill, and the vibration displacements were tested by the described sensors. The collection time was 60 seconds per sample. In this condition, 


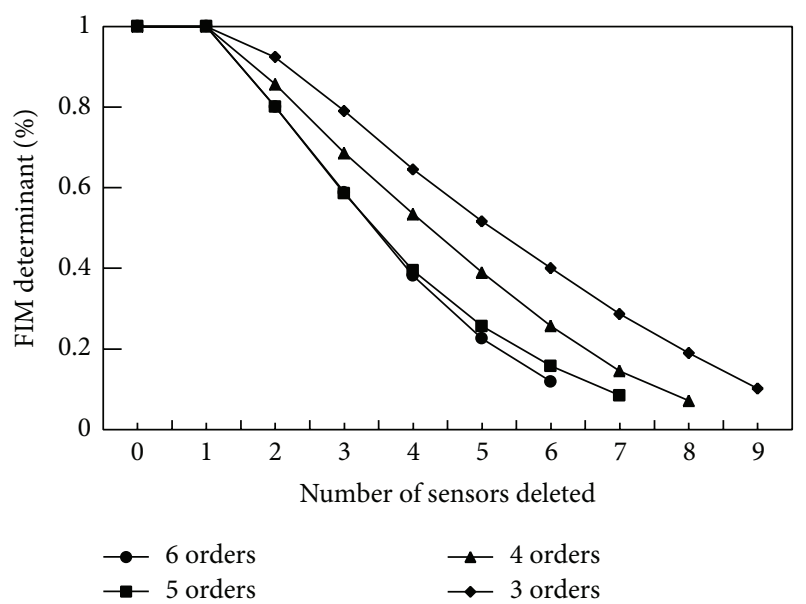

(a) $X$ direction

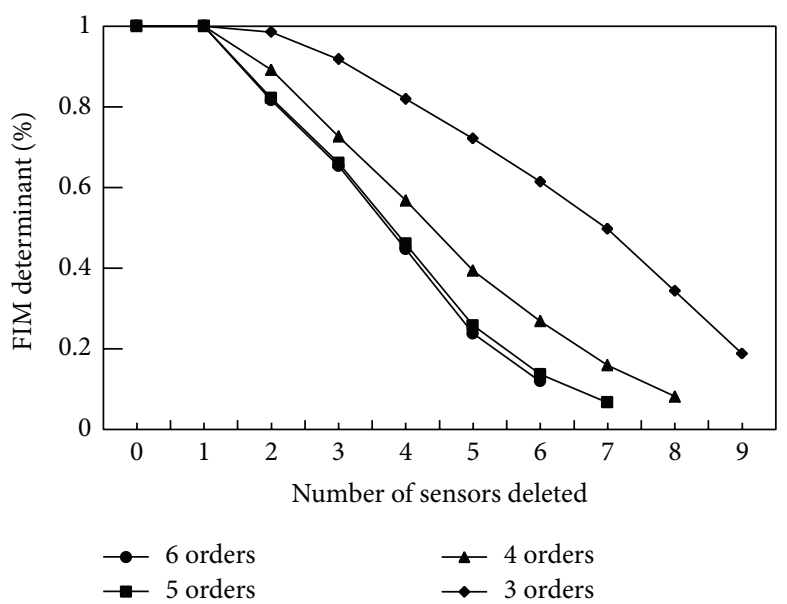

(b) $Y$ direction

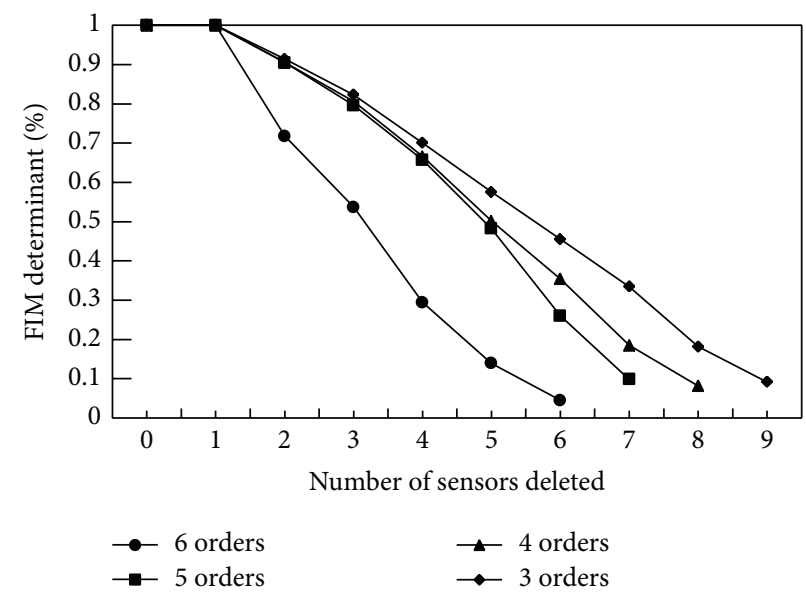

(c) $Z$ direction

FIGURE 5: Fisher information matrix determinant.

TABLE 4: Results of Fisher information matrix determinant.

\begin{tabular}{lcccccc}
\hline Direction & & \multicolumn{3}{c}{ Order } & \\
& $X(\mathrm{EfI})$ & $X(\mathrm{QR})$ & $Y(\mathrm{EfI})$ & $Y(\mathrm{QR})$ & $Z(\mathrm{EfI})$ & $2.61 \times 10^{-20}$ \\
\hline 5 & $3.53 \times 10^{-17}$ & $1.03 \times 10^{-17}$ & $7.17 \times 10^{-23}$ & $5.71 \times 10^{-23}$ & $1.24 \times 10^{-19}$ \\
4 & $2.49 \times 10^{-11}$ & $1.19 \times 10^{-11}$ & $4.28 \times 10^{-20}$ & $3.14 \times 10^{-20}$ & $8.60 \times 10^{-15}$ & $5.50 \times 10^{-15}$ \\
3 & $1.27 \times 10^{-8}$ & $1.17 \times 10^{-8}$ & $1.51 \times 10^{-13}$ & $1.09 \times 10^{-13}$ & $3.41 \times 10^{-9}$ & $1.35 \times 10^{-9}$ \\
\hline
\end{tabular}

the vibrations of the foundation and mill body were assumed to be the ergodic and stationary random processes; the collected samples can thus describe the vibration characteristics of the mill. The noise was eliminated using filter techniques with DASP software and the typical vibration curves for the mill obtained from the test were shown in Figure 10. It should be noted that only vibration displacement ranging from 0 to 10 second were shown in Figure 10.

4.3. Comparison of Vibration Displacement. Harmonic response analysis at the service load was performed by ANSYS. The analytical results of the vibration amplitude of each measuring point were compared with the test results, which was presented in Table 5. The test results in Table 5 were the maximum vibration displacements of these measuring points. The calculation results generally agreed with the field test results in $X$ direction, which indicated that the FE model could simulate the dynamic characteristics of the mediumspeed mill. It was also noted that the simulation in $Z$ and $Y$ directions was less effective than that in $X$ direction. From the location of measuring points, the test point 4 was more satisfactory, and the test points 2 and 3 were followed. Abundant dynamic characteristics of medium-speed mill were achieved based on the final sensors placement scheme, and the finite element analysis results were basically consistent with the field test results, which indicated the sensor placement optimization for vibration test on the mediumspeed mill was effective and economical. 


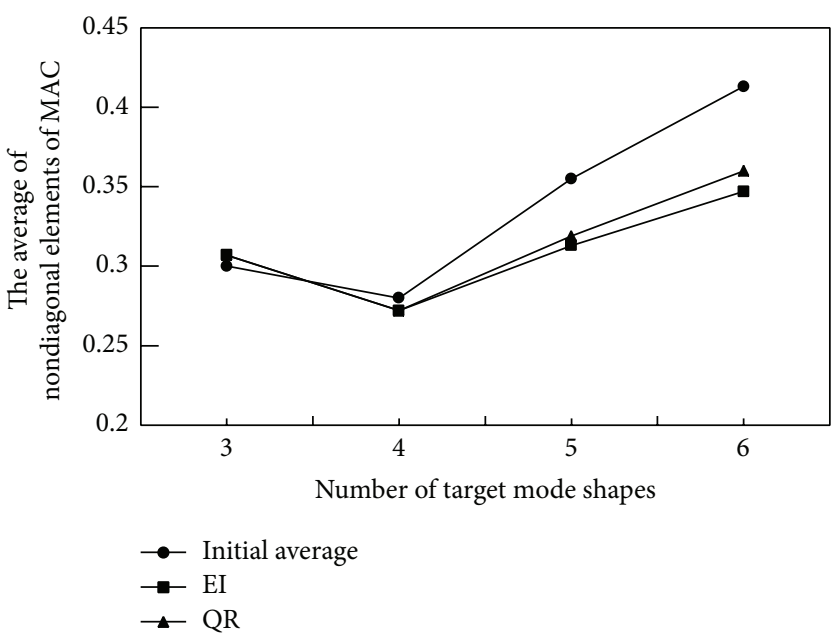

(a) $X$ direction

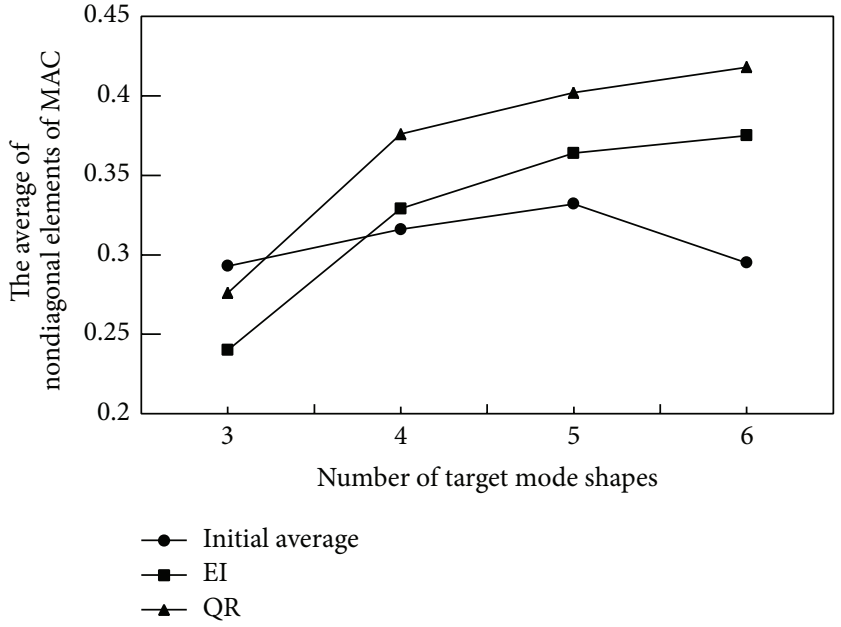

(b) $Y$ direction

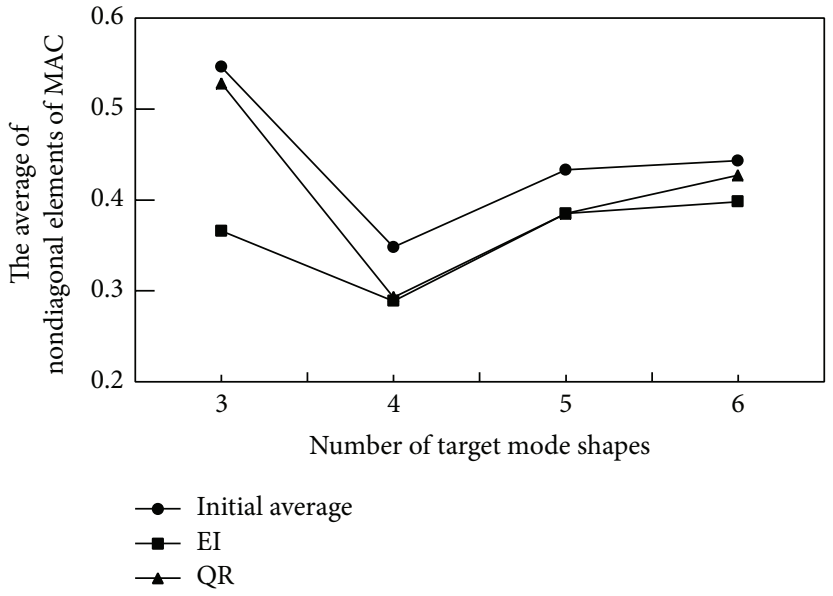

(c) $Z$ direction

FIGURE 6: Average values of MAC matrix off-diagonal elements.

TABLE 5: Comparison of vibration displacement between the finite element analysis results and the field test results.

\begin{tabular}{|c|c|c|c|c|c|c|c|}
\hline Measuring points & $2(X) / \mathrm{mm}$ & $3(X) / \mathrm{mm}$ & $4(X) / \mathrm{mm}$ & $2(Y) / \mathrm{mm}$ & $3(Y) / \mathrm{mm}$ & $4(Y) / \mathrm{mm}$ & $2(Z) / \mathrm{mm}$ \\
\hline Test results & 0.062 & 0.132 & 0.421 & 0.103 & 0.226 & 0.246 & 0.106 \\
\hline Calculation results & 0.051 & 0.101 & 0.385 & 0.080 & 0.169 & 0.202 & 0.082 \\
\hline Error (\%) & 17.5 & 23.5 & 8.6 & 22.4 & 25.1 & 18.3 & 22.6 \\
\hline
\end{tabular}

\section{Conclusion}

In this paper, the common methods and evaluation criteria for OSP had been summarized. The OSP of vibration test on medium-speed mill supported by spring vibration-isolated foundation was conducted using EfI and QR decomposition methods. It was found that these two methods for vibration test on medium-speed mill were all feasible, although this was different for some measuring points in different directions, and the difference became smaller with the decrease of the number of target mode shapes. For the evaluation criteria of FIM determinant, the effect was better in the case of small number of target mode shapes when the measuring points were certain; on the other hand, the effects in more target mode shapes were much more sensitive to the number of measuring points. For MAC, EfI method was more efficient than QR decomposition method. In addition, the OSP based on EfI method can achieve more efficient suboptimal measuring points.

The final scheme of OSP was based on the result that the first three mode shapes in $X$ direction were set as the targeted modal shapes. Six sensors were placed to achieve the dynamic response of the medium-speed mill, and the effect of the environmental and operational variability and noise contamination was reduced by filtering technique. The field test results were basically consistent with the calculation results by ANSYS, and the FEM of the medium-speed mill with spring vibration-isolated foundation could simulate 


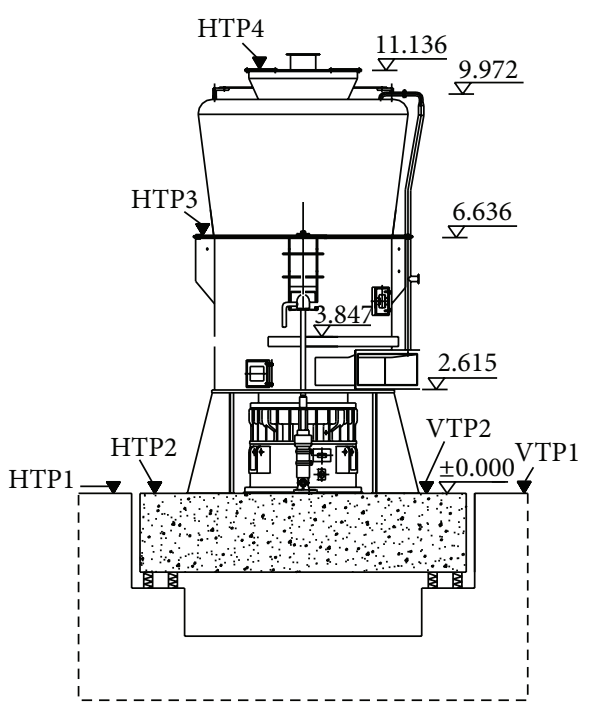

FIGURE 7: Placement of sensors (HTTP: horizontal test point, VTP: vertical test point).

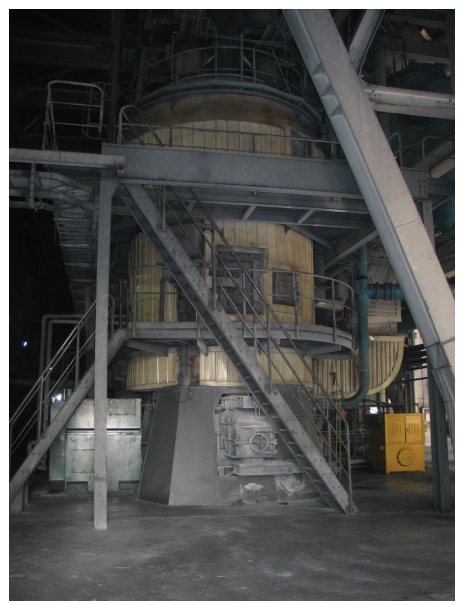

Figure 8: The elevation view of the tested medium-speed mill.

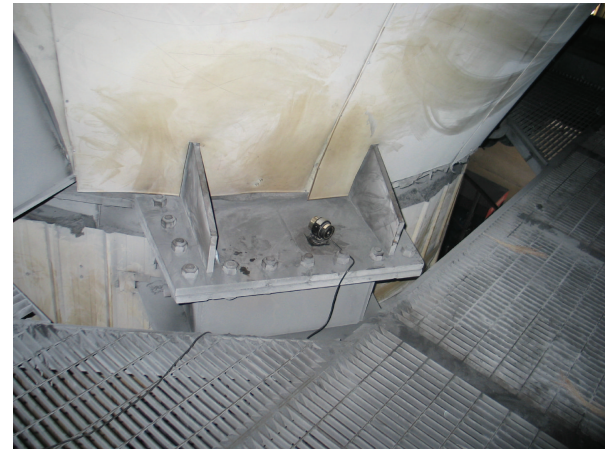

FIgURE 9: The sensor shown in close-up.
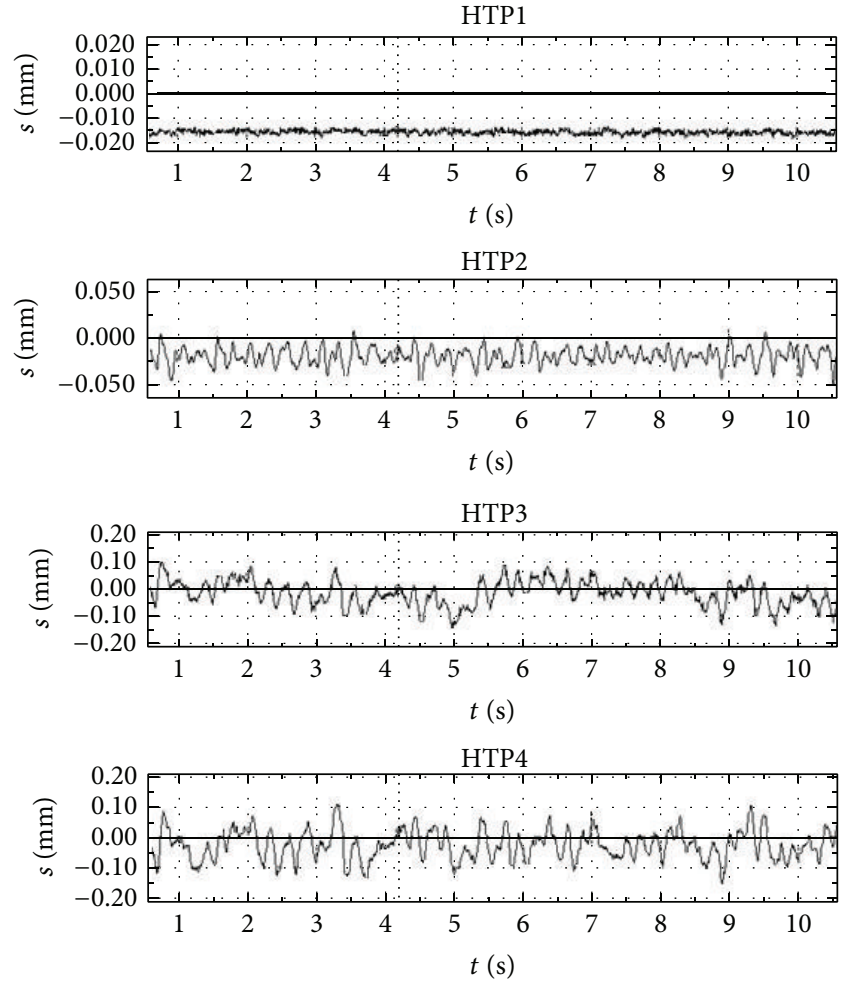

FIGURE 10: Horizontal vibration displacement in $X$ direction.

the vibration characteristic well. The sensor placement optimization for vibration test on the medium-speed mill was effective and economical.

\section{Conflict of Interests}

The authors declare that there is no conflict of interests regarding the publication of this paper.

\section{Acknowledgments}

The presented work was supported by the project of Shaanxi Province Excellent Young Scholars (2013KJXX-53), China Scholarship for Study Abroad, Program for Changjiang Scholars and Innovative Research Team in University, and Innovation Team of Xian University of Architecture and Technology.

\section{References}

[1] T. H. Yi, H. N. Li, and M. Gu, "Sensor placement optimisation for Dalian international trade mansion focusing on application demands," International Journal of Sensor Networks, vol. 15, no. 3, pp. 157-162, 2012.

[2] D. C. Kammer, "Sensor placement for on-orbit modal identification and correlation of large space structures," Journal of Guidance, Control, and Dynamics, vol. 14, no. 2, pp. 251-259, 1991.

[3] M. Papadopoulos and E. Garcia, "Sensor placement methodologies for dynamic testing," AIAA Journal, vol. 36, no. 2, pp. 256-263, 1998. 
[4] D. S. Li, H. N. Li, and C. P. Fritzen, "The connection between effective independence and modal kinetic energy methods for sensor placement," Journal of Sound and Vibration, vol. 305, no. 4-5, pp. 945-955, 2007.

[5] F. Cui, W. C. Yuan, and J. J. Shi, "Optimal sensor placement algorithms in bridge health monitoring," Journal of Tongji University, vol. 27, no. 2, pp. 165-169, 1999 (Chinese).

[6] H. M. Chow, H. F. Lam, T. Yin, and S. K. Au, "Optimal sensor configuration of a typical transmission tower for the purpose of structural model updating," Structural Control and Health Monitoring, vol. 18, no. 3, pp. 305-320, 2011.

[7] H. Wang, A. Li, T. Guo, and T. Tao, "Establishment and application of the wind and structural health monitoring system for the Runyang Yangtze River Bridge," Shock and Vibration, vol. 2014, Article ID 421038, 15 pages, 2014.

[8] L. H. Zhu, G. L. Bai, Y. L. Peng, F. J. Zhang, and X. L. Sun, "Vibration test and shock absorption research on medium-speed mill in thermal power plants," Journal of Building Structures, vol. 34, no. 5, pp. 43-51, 2013 (Chinese).

[9] T. G. Carne and C. R. Dohrmann, "A modal test design strategy for model correlation," in Proceedings of the 13th International Modal Analysis Conference, pp. 927-933, Nashville, Tenn, USA, February 1995.

[10] ANSYS Academic Research, Release 12.0.

[11] D. C. Kammer, "Sensor set expansion for modal vibration testing," Mechanical Systems and Signal Processing, vol. 19, no. 4, pp. 700-713, 2005.

[12] F. Moschas and S. C. Stiros, "Three-dimensional dynamic deflections and natural frequencies of a stiff footbridge based on measurements of collocated sensors," Structural Control and Health Monitoring, vol. 21, no. 1, pp. 23-42, 2014.

[13] P. Cornwell, C. R. Farrar, S. W. Doebling, and H. Sohn, "Environmental variability of modal properties," Experimental Techniques, vol. 23, no. 6, pp. 45-48, 1999.

[14] R. Castro-Triguero, S. Murugan, R. Gallego, and M. I. Friswell, "Robustness of optimal sensor placement under parametric uncertainty," Mechanical Systems and Signal Processing, vol. 41, no. 1-2, pp. 268-287, 2013. 

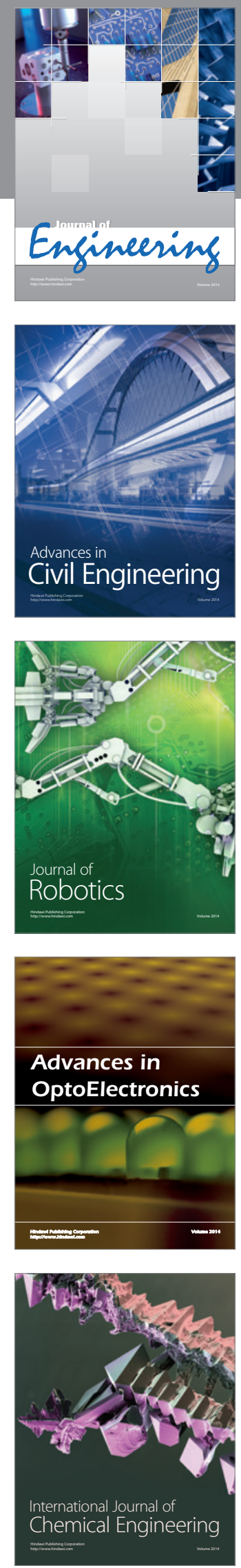

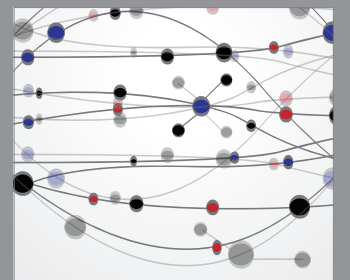

The Scientific World Journal
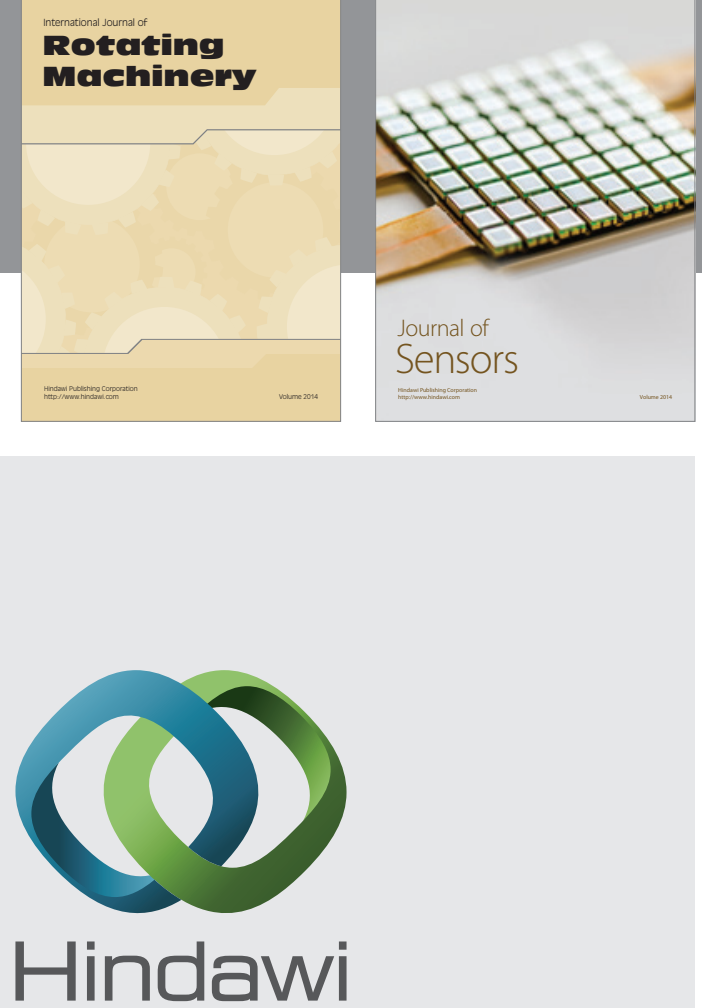

Submit your manuscripts at http://www.hindawi.com
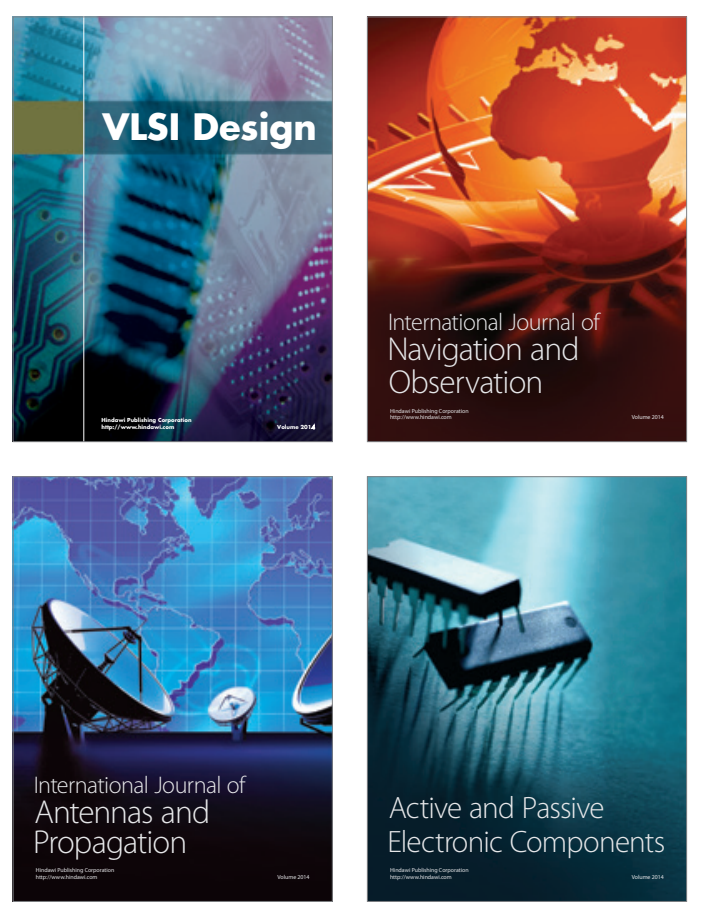
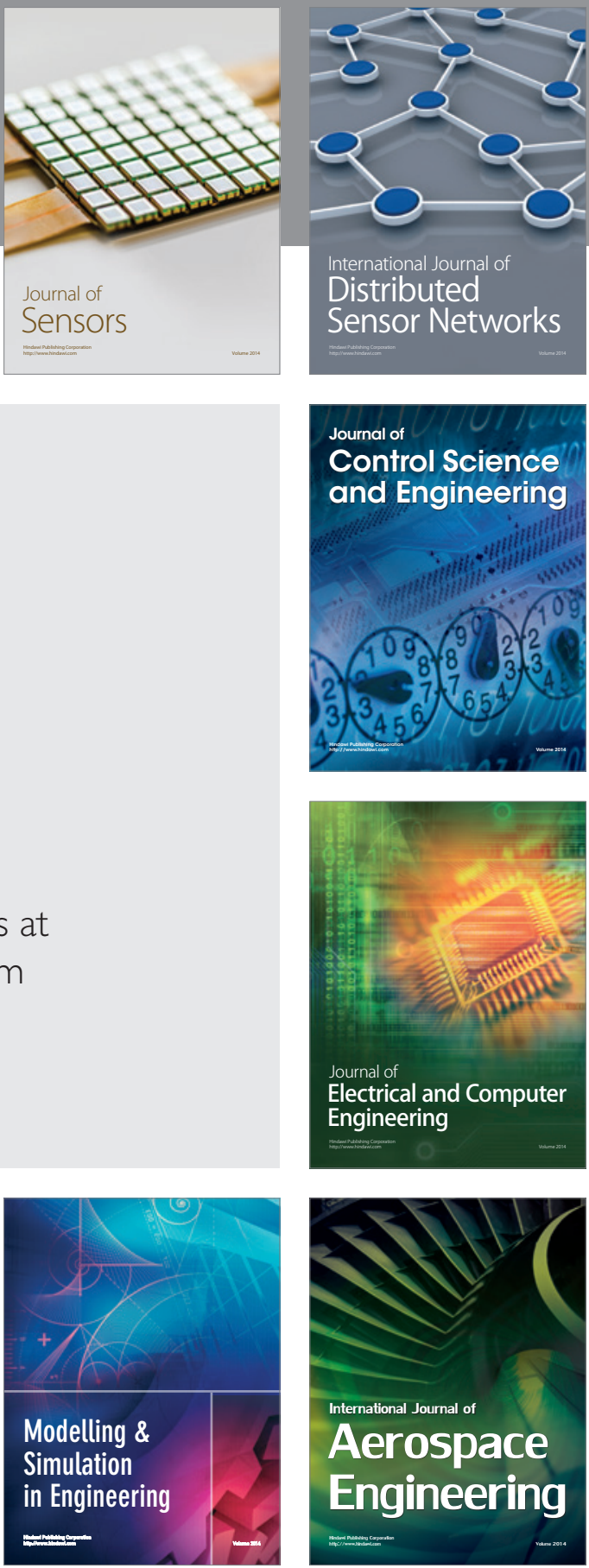

Journal of

Control Science

and Engineering
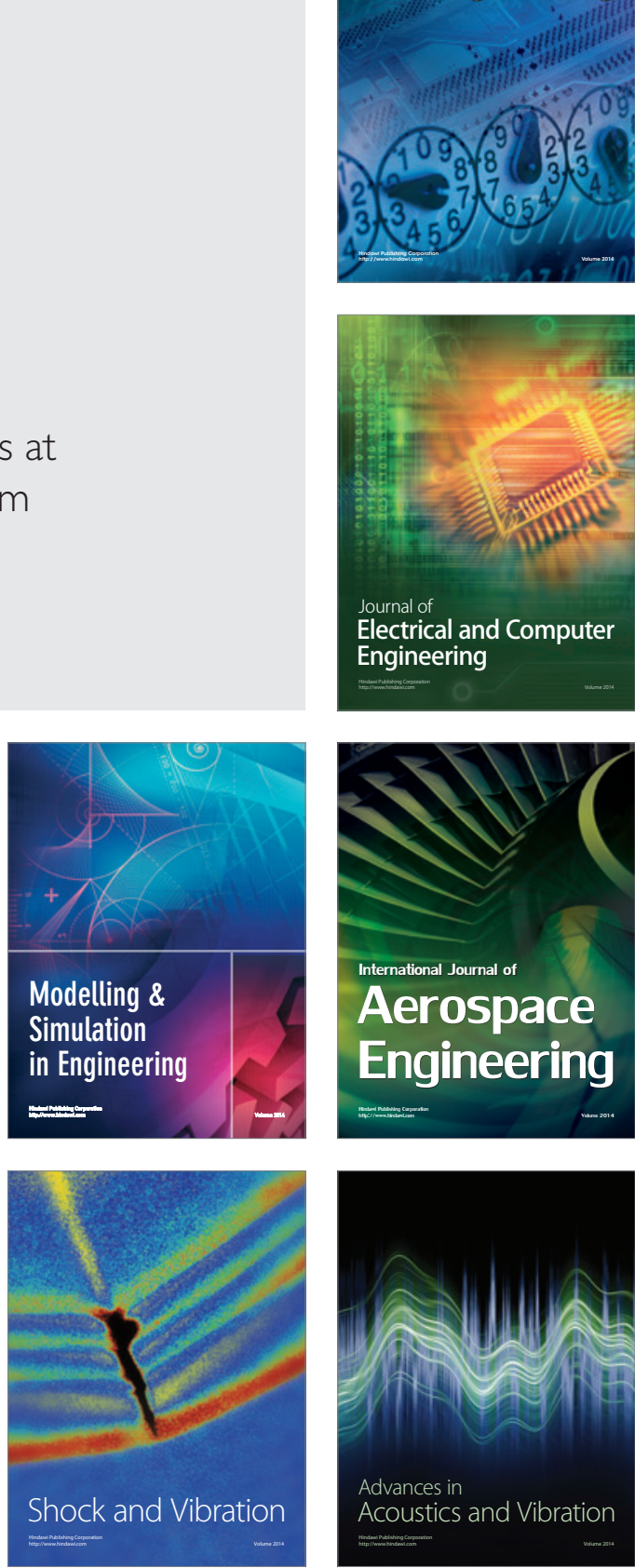